\title{
Expansion by Laguerre Function for Wave Diffraction around an Infinite Cylinder
}

\author{
Mingdong Lv', Hui Li' ${ }^{1}$, Huilong Ren ${ }^{1}$, Xiaobo Chen ${ }^{2}$ \\ ${ }^{1}$ College of Shipbuilding Engineering, Harbin Engineering University, Harbin, China \\ ${ }^{2}$ Bureau Veritas Research Department, Singapore City, Singapore \\ Email: 1213730780@qq.com
}

Received December 2014

\section{Abstract}

We consider a vertical circular cylinder on which the vertical variation of water diffraction waves is to be represented by a series of Laguerre functions $\ell_{n}(z)=e^{-z / 2} L_{n}(z)$ using Laguerre Polynomials $L_{n}(z)$. The variation is assumed to be of the form $1 /(1+z)^{n}$ with the integer $n$ depending on the radius of cylinder. Generally, the integer $n$ increases for a cylinder of larger diameter. The usual approximation by Laguerre functions is extended by introducing a scale parameter. The convergence of Laguerre series is then dependent on the value of the scale parameter $s$. The analytical and numerical computations of series coefficients are performed to study the number of series terms to keep the same accuracy. Indeed, the choice of integer $n$ depends on the scale parameter. Furthermore, diffraction waves generated by a semi-sphere inside the cylinder are evaluated on the cylinder surface. It is shown that the approximation by Laguerre series for diffraction waves on the cylinder is effective. This work provides important information for the choice of the radius of control surface in the domain decomposition method for solving hydrodynamic problems of body-wave interaction.

\section{Keywords}

Laguerre Function, Wave Diffraction around an Infinite Cylinder

\section{Introduction}

The Rankine source panel method needs a large number of panels due to panelizing the free surface as well as a damping zone avoiding the reflected wave from the sides of a numerical fluid domain. So a control surface can be introduced to divide the fluid domain into two subdomains by a control surface. This surface separates the problem into two problems: 1) the interior one in which the ship is of any form, the Green function is Rankine source Green function; 2) the exterior one in which the shape of the control surface is known and velocity potential is assumed to be known. It brings two important benefits: area to be discretized becomes smaller; no need to introduce the damping zone [1].

A circular cylinder is adopted as control surface. The vertical variation of water diffraction wave is assumed to be the form $1 /(1+z)^{n}$ which is represented by a series of Laguerre functions $\ell_{n}(z)$. Laguerre functions 
$\ell_{n}(z)$ which are defined by Laguerre polynomials $L_{n}(z)$ are a system of orthogonal functions on the interval $[0, \infty]$ [2]. It plays an important role in approximation and interpolation.

The purpose of this paper is to validate the accuracy and convergence of Laguerre series and approximate the vertical velocity potential $\phi$ on an infinite cylinder generated by a body. Section 2 introduces basis of Laguerre functions. Section 3 provides an analytical method to approximate the function $1 /(1+z)^{n}$ and the velocity potential $\phi$ by Laguerre functions. Section 4 uses some examples to investigate the convergence and accuracy of the method, and compares the result of Compass-Walcs-Basic.

\section{The Basis of Laguerre Function}

\subsection{Laguerre Polynomials}

The Laguerre polynomials are defined by the three-term recurrence relation

$$
\begin{gathered}
L_{0}(z)=1 \\
L_{1}(z)=1-z \\
L_{n}(z)=\frac{2 n-1-z}{n} L_{n-1}(z)-\frac{n-1}{n} L_{n-2}(z)
\end{gathered}
$$

$L_{n}(z)$ is called nth degree Laguerre polynomial [3].

The Laguerre polynomials have some useful relations

$$
\begin{gathered}
L_{n}(0)=1 \\
\frac{d L_{n}(0)}{d z}=-n \\
\frac{d L_{n}(z)}{d z}-\frac{d L_{n+1}(z)}{d z}=L_{n}(z)
\end{gathered}
$$

By virtu3 of Equation (6), we can obtain Equation (7)

$$
\frac{d\left[L_{n}(z)\right]}{d z}=-\sum_{k=0}^{n-1} L_{k}(z)
$$

We define $L_{n}^{(m+1)}(z)$ as Equation (8)

$$
\begin{aligned}
& \frac{d\left[L_{n}^{(m)}(z)\right]}{d z}=L_{n}^{(m+1)}(z) \quad m=0,1,2 \\
& \frac{d\left[L_{n-1}^{(m)}(z)\right]}{d z}-\frac{d\left[L_{n}^{(m)}(z)\right]}{d z}=L_{n-1}^{(m)}(z)
\end{aligned}
$$

By virtue of Equation (9), we can obtain Equation (10)

$$
\frac{d\left[L_{n}^{(m)}(z)\right]}{d z}=-\sum_{k=0}^{n-1} L_{k}^{(m)}(z)
$$

And the orthogonal relation

$$
\int_{0}^{\infty} L_{n}(z) L_{m}(z) e^{-z} d z=\delta_{n m}
$$

where $\delta_{n m}$ is Kronecker symbol.

Furthermore it can be easily shown that

$$
\int_{0}^{\infty} e^{-k z} L_{n}(z) d z=(k-1)^{n} / k^{n+1} \quad k \neq 0
$$

$k$ is a real number. 


\subsection{Laguerre Functions and Scale Parameter s}

We define nth degree Laguerre function $\ell_{n}(z)$ as:

$$
\ell_{n}(z)=e^{-z / 2} L_{n}(z)
$$

The Laguerre functions satisfy the orthogonal relation [3]:

$$
\int_{0}^{\infty} \ell_{n}(z) \ell_{m}(z)=\delta_{n m}
$$

where $\delta_{n m}$ is Kronecker symbol.

It is important to note that the Laguerre functions are well behaved. Indeed, the following properties are shown [3]:

$$
\left|\ell_{n}(z)\right| \leq 1 \quad \text { for } 0 \leq z \leq \infty
$$

and

$$
\ell_{n}(z)=\pi^{-1 / 2}(n z)^{-1 / 4} \cos (2 \sqrt{n z}-\pi / 4)+o\left(e^{-z / 2} n^{-3 / 4}\right)
$$

For $n=0,1,2$

We can approximate a function by a series of Laguerre functions with scale parameter $s$ :

$$
f(z)=\sum_{0}^{\infty} c_{n} \ell_{n}(s z)
$$

The Laguerre functions with scale parameter s also satisfy the orthogonal relation:

$$
\int_{0}^{\infty} \ell_{n}(s z) \ell_{m}(s z) d z=\frac{1}{s} \delta_{n m}
$$

The coefficient $c_{n}$ are defined

$$
c_{n}=\int_{0}^{\infty} f(z / s) \ell_{n}(z) d z
$$

\section{Numerical Approximation and Interpolation by Laguerre Functions}

As the velocity potential $\phi$ is assumed to $f(z)=1 /(1+z)^{n}$, we expand $f(z)=1 /(1+z)^{n}$ by Laguerre functions to validate the convergence and accuracy. In addition, we provide an interpolation method to approximate the velocity potential $\phi$.

For function $f(z)=1 /(1+z) \quad[4]$

$$
\begin{gathered}
f(z)=\frac{1}{1+z}=\sum_{n=0}^{\infty} c_{n} \ell_{n}(s z) \\
c_{n}=\int_{0}^{\infty}\left(1+\frac{z}{S}\right)^{-1} \ell_{n}(z) d z=\int_{0}^{\infty}\left(1+\frac{z}{S}\right)^{-1} e^{-z / 2} \ell_{n}(z) d z \\
c_{n}=\int_{0}^{\infty} e^{-x} d x \int_{0}^{\infty} e^{-(x / s+1 / 2) z} L_{n}(z) d z \\
c_{n}=\int_{0}^{\infty} e^{-x}\left(\frac{X}{S}-\frac{1}{2}\right)^{n} /\left(\frac{X}{S}+\frac{1}{2}\right)^{n+1} d x
\end{gathered}
$$

where we use the Equation (12) and Equation (24)

$$
1 /(1+z / s)=\int_{0}^{\infty} e^{-x(1+z / s)} d x
$$


The coefficient $c_{n}$ can be calculated by Equation (23) in Gauss-Laguerre integration, see in Equation (25)

$$
\int_{0}^{\infty} e^{-x} f(x) d x \approx \sum_{k=1}^{N} A_{k} f\left(x_{k}\right)
$$

where $x_{k}$ is the $k$ th distinct zero of $n$th Laguerre polynomial.

For function $f(z)=1 /(1+z)^{2}$

$$
\begin{gathered}
f(z)=\frac{1}{(1+z)^{2}}=\sum_{n=0}^{\infty} c_{n} \ell_{n}(s z) \\
c_{n}=\int_{0}^{\infty}(1+z / s)^{-2} \ell_{n}(z) d z=\int_{0}^{\infty} s^{2}(s+z)^{-2} \ell_{n}(z) d z \\
c_{n}=-s^{2}\left[\frac{\ell_{n}(z)^{\infty}}{z+s} \int_{0}^{\infty} \frac{1}{z+s} d \ell_{n}(z)\right] \\
c_{n}=s^{2}\left[\frac{1}{s}-\frac{1}{2} \int_{0}^{\infty} \frac{1}{z+s} e^{-z / 2} L_{n}(z) d z+\int_{0}^{\infty} \frac{1}{z+s} e^{-z / 2} \frac{d L_{n}(z)}{d z} d z\right]
\end{gathered}
$$

By virtue of Equation (7)

$$
c_{n}=s^{2}\left[\frac{1}{s}-\frac{1}{2} \int_{0}^{\infty} \frac{1}{z+s} e^{-z / 2} L_{n}(z) d x-\sum_{k=0}^{n-1} \int_{0}^{\infty} \frac{1}{z+s} e^{-z / 2} L_{k}(z) d z\right]
$$

Then the integration in Equation (30) can be calculated in Equation (21), $f(z)=1 /(1+z)^{n}$ can be expanded in the similar method. The $f(z)=1 /(1+z)^{3}$ is expanded by Laguerre functions as below

$$
c_{n}=\frac{s}{2}\left\{1+s\left[-\frac{2 n+1}{2}+\int_{0}^{\infty}\left(\frac{1}{4} e^{-z / 2} L_{n}(z)+\sum_{k=0}^{n-1} e^{-z / 2} L_{k}(z)+\sum_{k=1}^{n-1} \sum_{l=0}^{k-1} e^{-z / 2} L_{l}(z)\right) \frac{1}{z+s} d z\right]\right\}
$$

We suppose that the vertical velocity potential $\phi(z)$ is continuous for $z \geq 0$, where given function $\phi(z)$ is only known numerically at every point. The Laguerre-Gauss interpolation is applied to approximate the $\phi(z)$.

$$
\begin{gathered}
\phi(z)=\sum_{0}^{\infty} c_{n} \ell_{n}(z) \\
c_{n}=\int_{0}^{\infty} f(z) \ell_{n}(z) d z=\int_{0}^{\infty} e^{-\frac{z}{2}} f(z) L_{n}(z) d z \\
c_{n} \approx \sum_{k=0}^{N} A_{k} e^{\frac{z_{k}}{2}} f\left(z_{k}\right) L_{n}\left(z_{k}\right)
\end{gathered}
$$

\section{Numerical Results}

In this section, we present some numerical results. The algorithm is implemented by using Intel Visual Fortran Composer XE 2011 and all calculations are carried out in a computer of CPU $3.30 \mathrm{GHz}$.

We first use Equation (23) to approximate function $1 /(1+z)$, shown as Table 1. For description of the global errors, we introduce the notations. Approximation results by Laguerre functions use symbol $f_{a}(z)$.

$$
E_{r}=\left|\frac{f(z)-f_{a}(z)}{\max \{f(z)\}}\right|
$$

Then we use Equation (30) to approximate function $1 /(1+z)^{2}$, shown as Table 2.

At last, we use Equation (31) to approximate function $1 /(1+z)^{3}$ shown as Table 3.

A semi-sphere is adopted as a body inside the cylinder to generate diffraction waves. The radius of semisphere is $2 \mathrm{~m}$ as well as cylinder is $2.5 \mathrm{~m}$. The incident wave is in frequency of $0.6 \mathrm{rad} / \mathrm{s}$, in height of $2 \mathrm{~m}$. We prefer to approximate vertical variation with Equation (34) at $\theta$ is $0 \mathrm{rad} / \mathrm{s}$, shown in Figure 1. 
We compare the approximate results with results calculated by Compass-Walcs-Basic (CWB, a wave load software is developed by CCS), shown as Table 4.

\section{Conclusion}

In this note we presented a numerical method for interpolating vertical variation of water diffraction waves based on Laguerre functions. The convergence and accuracy is validated by approximating the functions

Table 1. Computed value and the relative errors at different values of $z$.

\begin{tabular}{|c|c|c|c|c|c|c|c|}
\hline \multirow{2}{*}{$\mathrm{z}$} & & $s=0.5, \mathrm{~N}=40$ & $s=0.5, \mathrm{~N}=40$ & $s=1.0, \mathrm{~N}=40$ & $s=1.0, \mathrm{~N}=40$ & $s=2.0, \mathrm{~N}=40$ & $S=2.0, \mathrm{~N}=40$ \\
\hline & $f(z)$ & $f_{a}(z)$ & $E_{r}$ & $f_{a}(z)$ & $E_{r}$ & $f_{a}(z)$ & $E_{r}$ \\
\hline 0 & $1.000 \mathrm{E}+00$ & $9.999 \mathrm{E}-01$ & $1.324 \mathrm{E}-04$ & $1.003 \mathrm{E}+00$ & $3.424 \mathrm{E}-03$ & $1.011 \mathrm{E}+00$ & $1.134 \mathrm{E}-02$ \\
\hline 1 & $5.000 \mathrm{E}-01$ & $5.000 \mathrm{E}-01$ & $4.161 \mathrm{E}-05$ & $5.006 \mathrm{E}-01$ & $6.478 \mathrm{E}-04$ & $5.000 \mathrm{E}-01$ & $1.167 \mathrm{E}-05$ \\
\hline 2 & $3.333 \mathrm{E}-01$ & $3.333 \mathrm{E}-01$ & $9.655 \mathrm{E}-06$ & $3.333 \mathrm{E}-01$ & $3.443 \mathrm{E}-06$ & $3.350 \mathrm{E}-01$ & $1.669 \mathrm{E}-03$ \\
\hline 3 & $2.500 \mathrm{E}-01$ & $2.500 \mathrm{E}-01$ & $1.329 \mathrm{E}-06$ & $2.495 \mathrm{E}-01$ & $4.639 \mathrm{E}-04$ & $2.509 \mathrm{E}-01$ & $8.635 \mathrm{E}-04$ \\
\hline 4 & $2.000 \mathrm{E}-01$ & $2.000 \mathrm{E}-01$ & $3.200 \mathrm{E}-05$ & $2.005 \mathrm{E}-01$ & $5.056 \mathrm{E}-04$ & $1.987 \mathrm{E}-01$ & $1.302 \mathrm{E}-03$ \\
\hline 30 & $3.22581 \mathrm{E}-02$ & $3.22278 \mathrm{E}-02$ & $3.02368 \mathrm{E}-05$ & $3.21187 \mathrm{E}-02$ & $1.39397 \mathrm{E}-04$ & $3.14553 \mathrm{E}-02$ & $8.02812 \mathrm{E}-04$ \\
\hline 40 & $2.43902 \mathrm{E}-02$ & $2.43811 \mathrm{E}-02$ & $9.10673 \mathrm{E}-06$ & $2.44373 \mathrm{E}-02$ & $4.70158 \mathrm{E}-05$ & $2.29822 \mathrm{E}-02$ & $1.40801 \mathrm{E}-03$ \\
\hline 50 & $1.96078 \mathrm{E}-02$ & $1.95427 \mathrm{E}-02$ & $6.51170 \mathrm{E}-05$ & $1.91617 \mathrm{E}-02$ & $4.46125 \mathrm{E}-04$ & $2.10475 \mathrm{E}-02$ & $1.43969 \mathrm{E}-03$ \\
\hline 60 & $1.63934 \mathrm{E}-02$ & $1.61789 \mathrm{E}-02$ & $2.14562 \mathrm{E}-04$ & $1.59564 \mathrm{E}-02$ & $4.37008 \mathrm{E}-04$ & $1.80588 \mathrm{E}-02$ & $1.66537 \mathrm{E}-03$ \\
\hline
\end{tabular}

Table 2. Computed value and the relative errors at different values of $\mathrm{z}$.

\begin{tabular}{|c|c|c|c|c|c|c|c|}
\hline \multirow{2}{*}{$\mathrm{z}$} & & $s=0.5, \mathrm{~N}=40$ & $s=0.5, \mathrm{~N}=40$ & $s=1.0, \mathrm{~N}=40$ & $s=1.0, \mathrm{~N}=40$ & $s=2.0, \mathrm{~N}=40$ & $S=2.0, \mathrm{~N}=40$ \\
\hline & $f(z)$ & $f_{a}(z)$ & $E_{r}$ & $f_{a}(z)$ & $E_{r}$ & $f_{a}(z)$ & $E_{r}$ \\
\hline 0 & $1.00000 \mathrm{E}+00$ & $9.97588 \mathrm{E}-01$ & $2.41169 \mathrm{E}-03$ & $9.99960 \mathrm{E}-01$ & $4.02065 \mathrm{E}-05$ & $1.00017 \mathrm{E}+00$ & $1.72137 \mathrm{E}-04$ \\
\hline 1 & $2.50000 \mathrm{E}-01$ & $2.50385 \mathrm{E}-01$ & $3.84964 \mathrm{E}-04$ & $2.49997 \mathrm{E}-01$ & $3.34900 \mathrm{E}-06$ & $2.50000 \mathrm{E}-01$ & $1.25300 \mathrm{E}-07$ \\
\hline 2 & $1.11111 \mathrm{E}-01$ & $1.10862 \mathrm{E}-01$ & $2.48952 \mathrm{E}-04$ & $1.11104 \mathrm{E}-01$ & $7.42851 \mathrm{E}-06$ & $1.11137 \mathrm{E}-01$ & $2.53938 \mathrm{E}-05$ \\
\hline 3 & $6.25000 \mathrm{E}-02$ & $6.27067 \mathrm{E}-02$ & $2.06697 \mathrm{E}-04$ & $6.24997 \mathrm{E}-02$ & $3.32780 \mathrm{E}-07$ & $6.25131 \mathrm{E}-02$ & $1.31245 \mathrm{E}-05$ \\
\hline 4 & $4.00000 \mathrm{E}-02$ & $3.98377 \mathrm{E}-02$ & $1.62343 \mathrm{E}-04$ & $4.00030 \mathrm{E}-02$ & $2.97746 \mathrm{E}-06$ & $3.99802 \mathrm{E}-02$ & $1.98408 \mathrm{E}-05$ \\
\hline 30 & $2.26757 \mathrm{E}-03$ & $1.01064 \mathrm{E}-03$ & $1.25694 \mathrm{E}-03$ & $1.03770 \mathrm{E}-03$ & $1.22987 \mathrm{E}-03$ & $1.02875 \mathrm{E}-03$ & $1.23883 \mathrm{E}-03$ \\
\hline 40 & $5.94884 \mathrm{E}-04$ & $5.63398 \mathrm{E}-04$ & $3.14860 \mathrm{E}-05$ & $5.99840 \mathrm{E}-04$ & $4.95552 \mathrm{E}-06$ & $5.76595 \mathrm{E}-04$ & $1.82893 \mathrm{E}-05$ \\
\hline 50 & $3.84468 \mathrm{E}-04$ & $3.64009 \mathrm{E}-04$ & $2.04587 \mathrm{E}-05$ & $3.87952 \mathrm{E}-04$ & $3.48407 \mathrm{E}-06$ & $4.16387 \mathrm{E}-04$ & $3.19192 \mathrm{E}-05$ \\
\hline 60 & $2.68745 \mathrm{E}-04$ & $2.61505 \mathrm{E}-04$ & $7.24000 \mathrm{E}-06$ & $2.80978 \mathrm{E}-04$ & $1.22328 \mathrm{E}-05$ & $3.13363 \mathrm{E}-04$ & $4.46180 \mathrm{E}-05$ \\
\hline
\end{tabular}

Table 3. Computed value and the relative errors at different values of $z$.

\begin{tabular}{cccccccc}
\hline \multirow{2}{*}{$\mathrm{z}$} & & $s=0.5, \mathrm{~N}=40$ & $s=0.5, \mathrm{~N}=40$ & $s=1.0, \mathrm{~N}=40$ & $s=1.0, \mathrm{~N}=40$ & $s=2.0, \mathrm{~N}=40$ & $S=2.0, \mathrm{~N}=40$ \\
\cline { 2 - 8 } & $f(\mathrm{z})$ & $f_{a}(\mathrm{z})$ & $E_{r}$ & $f_{a}(\mathrm{z})$ & $E_{r}$ & $f_{a}(\mathrm{z})$ & $E_{r}$ \\
\hline 0 & $1.00000 \mathrm{E}+00$ & $9.92900 \mathrm{E}-01$ & $7.10008 \mathrm{E}-03$ & $9.99653 \mathrm{E}-01$ & $3.46592 \mathrm{E}-04$ & $9.99998 \mathrm{E}-01$ & $2.05300 \mathrm{E}-06$ \\
1 & $1.25000 \mathrm{E}-01$ & $1.26088 \mathrm{E}-01$ & $1.08848 \mathrm{E}-03$ & $1.24953 \mathrm{E}-01$ & $4.66923 \mathrm{E}-05$ & $1.25000 \mathrm{E}-01$ & $3.33900 \mathrm{E}-07$ \\
2 & $3.70370 \mathrm{E}-02$ & $3.63692 \mathrm{E}-02$ & $6.67851 \mathrm{E}-04$ & $3.70089 \mathrm{E}-02$ & $2.81209 \mathrm{E}-05$ & $3.70370 \mathrm{E}-02$ & $1.40070 \mathrm{E}-08$ \\
3 & $1.56250 \mathrm{E}-02$ & $1.61809 \mathrm{E}-02$ & $5.55894 \mathrm{E}-04$ & $1.56479 \mathrm{E}-02$ & $2.28585 \mathrm{E}-05$ & $1.56249 \mathrm{E}-02$ & $1.12660 \mathrm{E}-07$ \\
4 & $8.00000 \mathrm{E}-03$ & $7.55455 \mathrm{E}-03$ & $4.45448 \mathrm{E}-04$ & $7.98476 \mathrm{E}-03$ & $1.52402 \mathrm{E}-05$ & $7.99978 \mathrm{E}-03$ & $2.17222 \mathrm{E}-07$ \\
30 & $3.35672 \mathrm{E}-05$ & $-4.74463 \mathrm{E}-05$ & $8.10135 \mathrm{E}-05$ & $2.90084 \mathrm{E}-05$ & $4.55883 \mathrm{E}-06$ & $3.33679 \mathrm{E}-05$ & $1.99241 \mathrm{E}-07$ \\
40 & $1.45094 \mathrm{E}-05$ & $-7.74084 \mathrm{E}-05$ & $9.19178 \mathrm{E}-05$ & $1.83909 \mathrm{E}-05$ & $3.88152 \mathrm{E}-06$ & $1.41008 \mathrm{E}-05$ & $4.08583 \mathrm{E}-07$ \\
50 & $7.53858 \mathrm{E}-06$ & $-7.10022 \mathrm{E}-05$ & $7.85408 \mathrm{E}-05$ & $7.11794 \mathrm{E}-06$ & $4.20641 \mathrm{E}-07$ & $7.37819 \mathrm{E}-06$ & $1.60386 \mathrm{E}-07$ \\
60 & $4.40566 \mathrm{E}-06$ & $-5.84196 \mathrm{E}-05$ & $6.28252 \mathrm{E}-05$ & $1.67154 \mathrm{E}-06$ & $2.73412 \mathrm{E}-06$ & $4.30944 \mathrm{E}-06$ & $9.62135 \mathrm{E}-08$ \\
\hline
\end{tabular}


Table 4. Computed value and the relative errors at different values of $z$.

\begin{tabular}{ccccccc}
\hline \multirow{2}{*}{$\mathrm{z}$} & \multicolumn{3}{c}{ Velocity potential (real part) } & \multicolumn{2}{c}{ Velocity potential (imaginary part) } \\
\cline { 2 - 7 } & $\phi_{\text {CWB }}(\mathrm{z})$ & $\phi_{\text {LAG }}(\mathrm{z})$ & $\mathrm{E}_{r}$ & $\phi_{\text {CWB }}(\mathrm{z})$ & $\phi_{\text {LAG }}(\mathrm{z})$ & $E_{r}$ \\
\hline 0 & $-6.88127 \mathrm{E}-02$ & $-6.95262 \mathrm{E}-02$ & $1.02611 \mathrm{E}-02$ & $-2.24248 \mathrm{E}-04$ & $-2.21099 \mathrm{E}-04$ & $1.40408 \mathrm{E}-02$ \\
1 & $-6.61136 \mathrm{E}-02$ & $-6.60080 \mathrm{E}-02$ & $1.51998 \mathrm{E}-03$ & $-1.83157 \mathrm{E}-04$ & $-1.83624 \mathrm{E}-04$ & $2.08272 \mathrm{E}-03$ \\
2 & $-6.35524 \mathrm{E}-02$ & $-6.34498 \mathrm{E}-02$ & $1.47629 \mathrm{E}-03$ & $-1.48078 \mathrm{E}-04$ & $-1.48531 \mathrm{E}-04$ & $2.01776 \mathrm{E}-03$ \\
3 & $-6.11155 \mathrm{E}-02$ & $-6.12360 \mathrm{E}-02$ & $1.73354 \mathrm{E}-03$ & $-1.18161 \mathrm{E}-04$ & $-1.17630 \mathrm{E}-04$ & $2.37039 \mathrm{E}-03$ \\
4 & $-5.87918 \mathrm{E}-02$ & $-5.86911 \mathrm{E}-02$ & $1.44937 \mathrm{E}-03$ & $-9.26525 \mathrm{E}-05$ & $-9.30975 \mathrm{E}-05$ & $1.98463 \mathrm{E}-03$ \\
5 & $-5.65723 \mathrm{E}-02$ & $-5.66178 \mathrm{E}-02$ & $6.54045 \mathrm{E}-04$ & $-7.08996 \mathrm{E}-05$ & $-7.07011 \mathrm{E}-05$ & $8.85062 \mathrm{E}-04$ \\
10 & $-4.68030 \mathrm{E}-02$ & $-4.67179 \mathrm{E}-02$ & $1.22372 \mathrm{E}-03$ & $-1.67622 \mathrm{E}-06$ & $-2.05020 \mathrm{E}-06$ & $1.66772 \mathrm{E}-03$ \\
20 & $-3.22544 \mathrm{E}-02$ & $-3.21762 \mathrm{E}-02$ & $1.12523 \mathrm{E}-03$ & $4.18505 \mathrm{E}-05$ & $4.15041 \mathrm{E}-05$ & $1.54439 \mathrm{E}-03$ \\
30 & $-2.23033 \mathrm{E}-02$ & $-2.23401 \mathrm{E}-02$ & $5.28384 \mathrm{E}-04$ & $4.47875 \mathrm{E}-05$ & $4.49518 \mathrm{E}-05$ & $7.32494 \mathrm{E}-04$ \\
40 & $-1.54425 \mathrm{E}-02$ & $-1.53552 \mathrm{E}-02$ & $1.25602 \mathrm{E}-03$ & $3.80591 \mathrm{E}-05$ & $3.76742 \mathrm{E}-05$ & $1.71664 \mathrm{E}-03$ \\
50 & $-1.06995 \mathrm{E}-02$ & $-1.06060 \mathrm{E}-02$ & $1.34520 \mathrm{E}-03$ & $2.99432 \mathrm{E}-05$ & $2.95298 \mathrm{E}-05$ & $1.84357 \mathrm{E}-03$ \\
60 & $-7.41677 \mathrm{E}-03$ & $-7.33185 \mathrm{E}-03$ & $1.22143 \mathrm{E}-03$ & $2.27477 \mathrm{E}-05$ & $2.23748 \mathrm{E}-05$ & $1.66273 \mathrm{E}-03$ \\
\hline
\end{tabular}

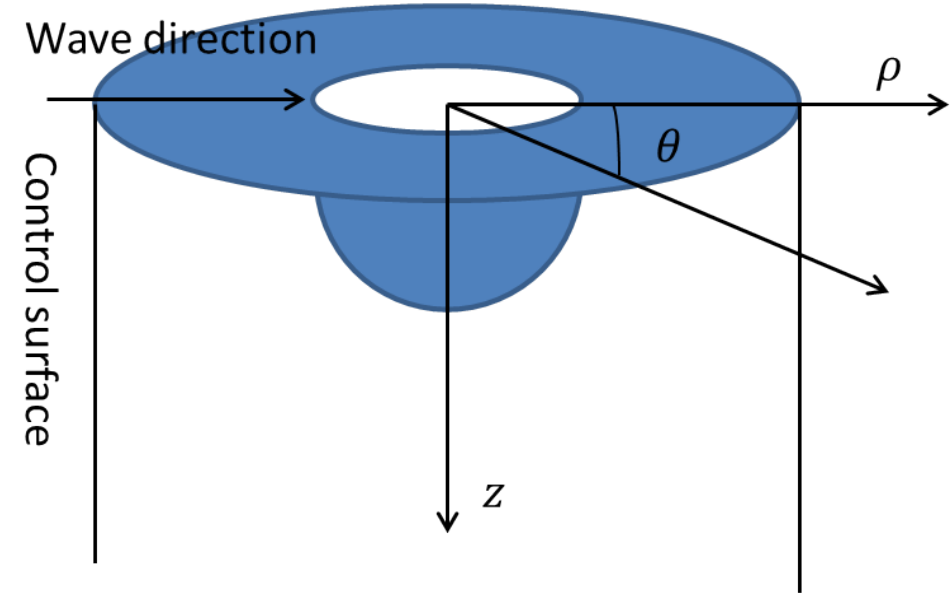

Figure 1. A semi-sphere in a cylinder.

$1 /(1+z)^{n} \quad(n=0,1,2 \cdots)$. It is applicable to approximate the vertical variation around a circle cylinder by Laguerre functions.

\section{References}

[1] Ten, I. and Chen, X.B. (2011) Zero Speed Rankine-Kelvin Hybrid Method. Bureau Veritas Research Department, 6.

[2] Guo, B.-Y. and Wang, Z.-Q. (2007) Numerical Integration Based on Laguerre-Gauss interpolation. Comput. Methods Appl. Mech. Engrg, 196, 3726-3741. http://dx.doi.org/10.1016/j.cma.2006.10.035

[3] Abramowitz, M. and Stegun, I.A. (1967) Handbook of Mathematical Functions. Dover Publications.

[4] Keilson, J., Nunn, W. and Sumita, U. (1980) The Laguerre Transform. Center for Naval Analysys, 26-28. 